\title{
Internal Hernia After Gastric Bypass: A New and Simplified Technique for Laparoscopic Primary Closure of the Mesenteric Defects
}

\author{
Ebrahim Aghajani • Hedin J. Jacobsen • \\ Bent Johnny Nergaard • Jan L. Hedenbro • \\ Björn Geir Leifson • Hjörtur Gislason
}

Received: 7 October 2011 / Accepted: 11 November 2011 /Published online: 29 November 2011

(C) The Author(s) 2011. This article is published with open access at Springerlink.com

\begin{abstract}
Background Bowel obstruction due to internal hernia is a well-known complication of laparoscopic Roux-en-Y gastric bypass (LRGB). Increasing evidence supports primary closing of the mesenteric defects, but controversy continues about surgical technique of systematic closure. This paper reviews our experience with internal hernia after LRGB and describes a new method of preemptive closure of the mesenteric defects.

Material and Methods Two thousand four hundred seventy-two consecutive patients undergoing LRGB from September 2005 to June 2010 were entered into our prospective longitudinal database. The mesenteric defects were not closed. Patients entered a 5-year follow-up program, and all who subsequently presented with internal hernia were analyzed. A further 1,630 patients operated on in the last 12 months were subjected to our new technique of closing the defects; data were entered in our own database as well as in the Scandinavian quality registry. Follow-up time for these patients is limited.

Results In the first group, 117 patients developed an internal hernia (4.7\%) at a mean interval after LRGB of 13 (range, 4-43 months). Four patients needed bowel resections because of severe ischemia. There was one death associated with complication of the internal hernia. In the primary closure group, four patients early in the series had reoperations for kinking of the enteroanastomosis. There have been no mesenteric haematomas encountered.

Conclusions Internal hernia should be ruled out in patients with previous LRGB and abdominal pain. Our technique for primary closing of the mesenteric defects seems to be safe and is so far promising.
\end{abstract}

Keywords Internal hernia $\cdot$ LRGB $\cdot$ Bowel obstruction • Mesenteric defects

This paper was partly presented at IFSO 2011, 1 September 2011, Hamburg.

E. Aghajani $(\bowtie) \cdot$ H. J. Jacobsen $\cdot$ B. J. Nergaard $\cdot$ H. Gislason Department of Surgery, Aleris Hospital,

Fredrik-Stangsgt. 11-13,

0264 Oslo, Norway

e-mail: Ebrahim.Aghajani@aleris.no

J. L. Hedenbro • B. G. Leifson

Aleris Obesitas Skåne,

Skåne, Sweden

\section{Introduction}

The laparoscopic Roux-en-Y gastric bypass (LRGB) is currently the preferred procedure in the management of severely obese patients. With increasing numbers of operated patients, late complications will be more apparent. Internal hernias are the main cause of late postoperative morbidity, causing abdominal discomfort and sometimes leading to bowel obstruction.

The reported incidence of internal hernias varies. We present a large series from a single team of surgeons with a high internal follow-up rate.

The closure of mesenteric defects involves the suturing or other manipulation of the small bowel mesentery with 
the risk of haematomas and disturbed anatomy of the enteroanastomosis.

Against the background of our previous experience, we wanted to test whether a new, simple approach to closing the mesenteric defects could be introduced without a high cost in short-term complications.

\section{Patient Material}

Aleris Hospital in Oslo, Norway, and Aleris Obesitas Skåne in Kristianstad, Sweden, are private clinics with dedicated bariatric units, using the same rotating team of surgeons. Patient demographics are summarised in Table 1. Indications for surgery were the same as generally accepted as a good standard for bariatric surgery (European Guidelines).

Previous Policy During the period from September 2005 until June 2010, we performed, in total, 2,472 LRGB without closing the mesenteric defects.

New Policy From 1 June 2010 to 1 June 2011, we performed 1,630 LRGB and closed the mesenteric defect in all our patients with our new technique.

\section{Operative Methods}

Five ports were used: two $5-\mathrm{mm}$ and two $12-\mathrm{mm}$ ports for instruments and one 10-mm for the camera (Fig. 1). Our surgical procedure has been described in more detail earlier. $^{1}$

The first part of the operation was the same in all cases. A very small gastric pouch was created and the bowel brought up, first as an omega loop in an antecolic and antegastric fashion. Following linear stapling of the anastomoses and division of the omega loop, the last step was testing the integrity of the gastrojejunostomy by inflating it with methylene-blue-dyed saline via a nasogastric tube.

Table 1 Patient demographics

\begin{tabular}{lll}
\hline & 2005-May 2010 & June 2010-June 2011 \\
\hline Total number of patients & 2,472 & 1,630 \\
Age at operation & $42(17-74)$ & $41(17-69)$ \\
BMI at operation & $43(29-68)$ & $42(29-62)$ \\
FU time (months) & $23(7-64)$ & a \\
\hline
\end{tabular}

${ }^{\mathrm{a}}$ Follow-up at the time of writing insufficient for analysis

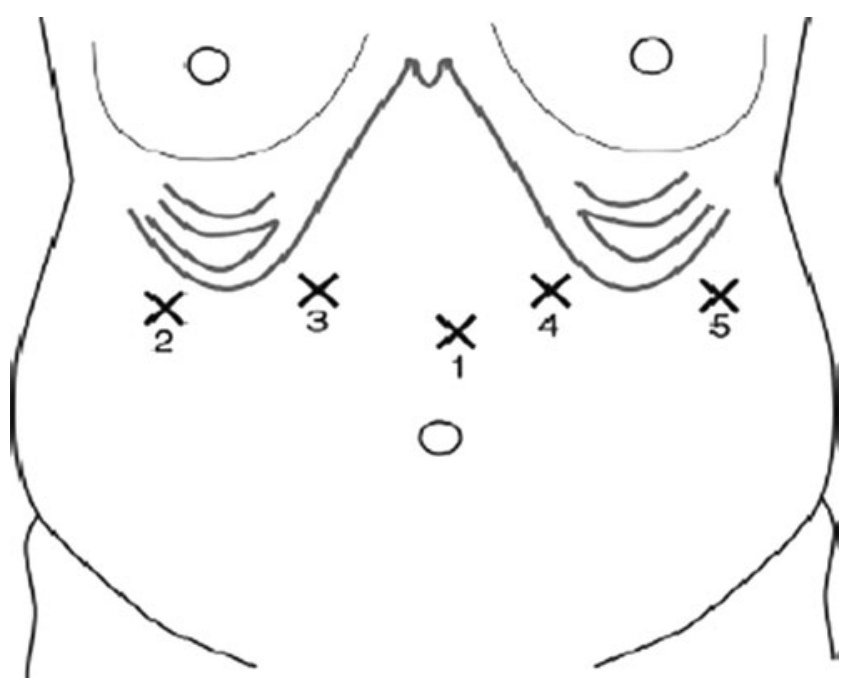

Fig. 1 Position of trocars for gastric bypass

Technique for Primary Laparoscopic Closing of Mesenteric Defects

The closing procedure was started after dividing the omega loop, with the patient still in the reverse Trendelenburg position.

Petersen's Defect The Endo Universal ${ }^{\mathrm{TM}} 4.8 \mathrm{~mm}$ stapler (Autosuture) was inserted via trocar \#4 $(12 \mathrm{~mm})$. With gentle manoeuvres, a pair of graspers was used to expose the Petersen's defect by lifting the transverse mesocolon (Fig. 2). The staples were partially extended presenting 'hooks' that facilitated the catching and adaptation of the mesenteric serosa. We closed the Petersen's defect from the root of the mesentery of the Roux limb and transverse colon up to the transverse colon itself. To avoid vascular injury, care was taken not to press the clip-applicator too deeply.



Fig. 2 Closure of Petersen's space 
Jejunal Mesenteric Defect The assistant pulled up the end of the duodenal limb in order to expose properly this mesenteric defect as shown in Fig. 3. We used the same type of laparoscopic stapler via trocar \#4 also for the closing of this defect. We took care to avoid kinking of the Roux-limb at level of the jejuno-jejunostomy.

\section{Statistics}

Data from all patients during the study period were prospectively collected in a proprietary database. Statistical analysis was performed using the appropriate programs of SPSS system, v. 19.0. All data are presented as median and range, unless otherwise stated.

\section{Results}

\section{Non-closure of Mesenteric Defects}

We performed 2,472 LRGB in our two hospitals between September 2005 and June 2010 (Table 1).

One hundred seventeen patients (100 women and 17 men) with a mean age of 41 years (range, 22-67 years) developed an internal hernia (4.7\%) at a mean interval after LRGB of 13 (range, 4-43 months). The mean BMI the time of internal hernia was 27 (range, $18-38 \mathrm{~kg} / \mathrm{m}^{2}$ ). In 46 (39\%) cases, the internal hernia was at the jejunojejunostomy; 28 (24\%) cases had an internal hernia at Petersen's defect. Forty-three patients were found only to have a mesenteric defect without overt herniation of bowel. The latter is consistent with an intermittent internal hernia that reduces itself spontaneously.

Ninety-nine of the patients (85\%) were admitted to our hospitals. The diagnosis of internal herniation was con-

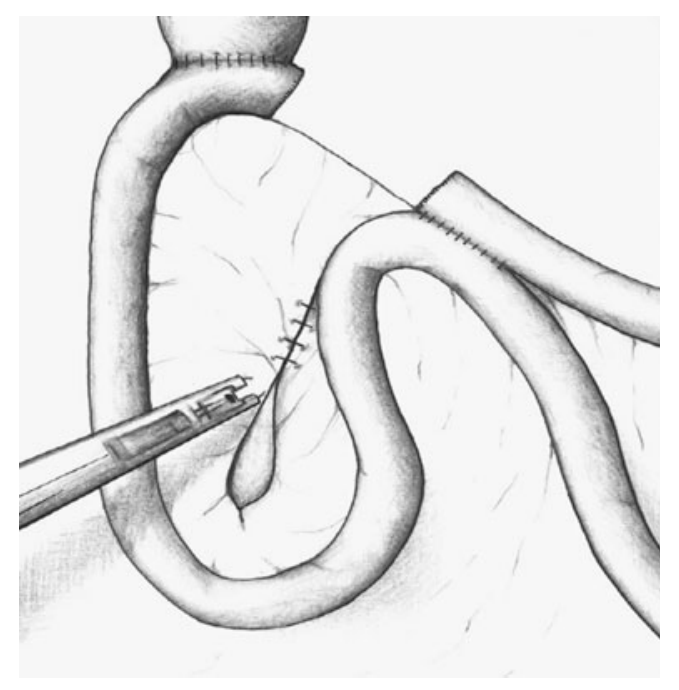

Fig. 3 Closure of jejunal mesenteric defect

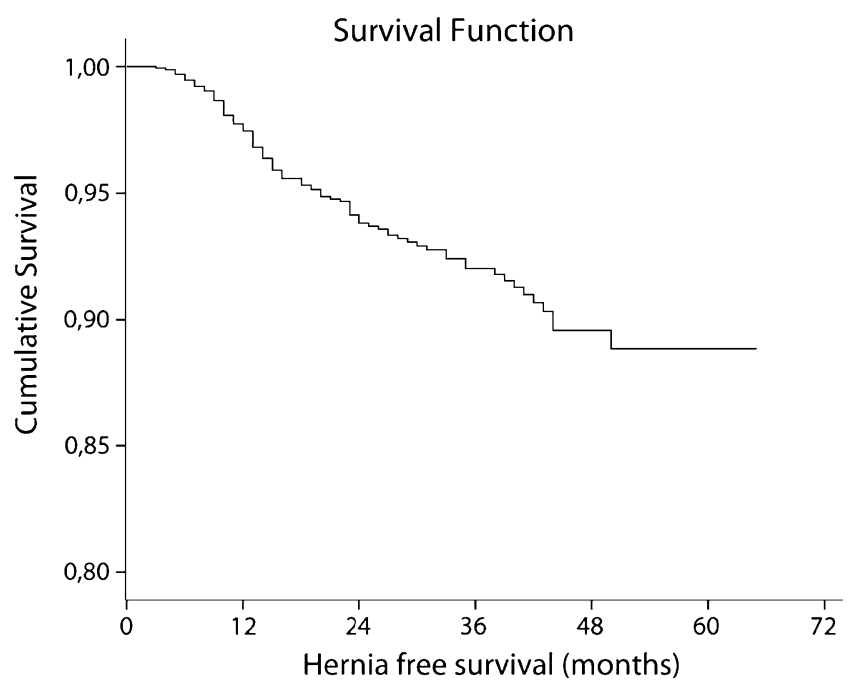

Fig. 4 A survival function diagram drawn using the life-table method demonstrates well the relation of internal hernia occurrence to postoperative time. Operation for internal hernia is the terminal event. The analysis is based on 1,671 patients operated in Oslo with LRGB from 2006 to mid 2010 . One hundred four $(6.2 \%$ overall $)$ cases had been reoperated for confirmed, clinically significant internal hernia. This diagram illustrates how the internal hernia cases start to occur 3 months post-operatively and seem to be uniformly frequent through the first 3 years. Internal hernia operation-free survival by this method is estimated to be $88.9 \%$ at the end of a 5 -year follow-up

firmed laparoscopically and the reduction of the hernia and closure of the mesenteric defects undertaken with a nonabsorbable running suture. No patient needed bowel resection. Two patients were pregnant in the third trimester. The remaining 18 patients were admitted acutely to other hospitals. Of them, seven were operated on by open surgery; four patients needed bowel resections $(70-300 \mathrm{~cm})$ because of severe ischemia. One patient died of septic complications associated with internal hernia at an outside hospital.

A life-table analysis was performed, which estimates an $11.1 \%$ 5-year incidence of clinically significant internal hernia requiring operation (Fig. 4).

\section{Closure of Mesenteric Defects by Our New Technique}

Between June 2010 and June 2011, we operated 1,630 consecutive LRGB patients where the mesenteric defects were closed using the EndoHernia stapling device (Endo Universal $4.8 \mathrm{~mm}$, Autosuture).

Stopwatch timing in 20 cases showed an average time used for closing both mesenteric defects of $1 \mathrm{~min}$ and $49 \mathrm{~s}$ (1:20 to $2: 50)$.

Four patients $(0.2 \%)$ in the group with mesenteric closure have been re-operated on because of bowel obstruction at the entero-anastomosis directly related to the closure of this mesenteric defect. All were early in the series and operated within 4 days of the primary operation. Twenty-two patients operated by this method have been re- 
operated on later for other reasons, and in all cases, the two mesenteric defects were examined and complete closure ascertained and documented (Fig. 5).

\section{Discussion}

The clinical presentation of internal hernia ranges from intermittent pain, often in the left upper abdomen through more constant abdominal pain, with or without nausea and vomiting to severe, acute abdominal pain. ${ }^{2}$ A certain diagnosis can most often only be made by laparoscopy. In case of uncertain abdominal pain in patients with LRGB, our policy is to perform gastroscopy to exclude pouch ulcers and ultrasound examination, revealing cholecystolithiasis. Whenever a laparoscopy is indicated in these patients, mesenteric defects should be closed concomitantly. Internal hernia may often co-exist with ulcer or gallstones, which can lead to diagnostic error. A high suspicion of internal hernia is in order, even in the presence of other possible causes of pain such as pouch-ulcer.

It seems that the laparoscopic approach is associated with a higher risk of internal hernias than that of open gastric bypass, due to less postoperative adhesions. The reported incidence of internal hernia after LRGB ranges widely, from $0.2 \%$ to $9 \% .^{2-7}$ The incidence also depends on the surgical method, with the retrocolic route of the alimentary-limb causing more internal hernias than the antecolic route. ${ }^{4,8}$

Primary closure of the mesenteric defects seems to reduce the incidence of internal hernia ${ }^{5}$, but can also be associated with complications. By suturing the mesentery, haematomas can occur, causing circulation impairment to the intestine. Closure of the mesenteric defects can also result in increased rate of obstruction at the entero-anastomosis caused by adhesion or rotation of the anastomosis. ${ }^{9}$

Our present cumulative incidence of internal herniation of $4.7 \%$ lies within the published limits. ${ }^{7,10}$ However, we are convinced this incidence is underestimated because the duration of the follow-up is still limited. A lifetime risk in excess of the estimated 5 -year incidence of $11.1 \%$ is what can be expected by a longer follow-up (Fig. 4).

Internal hernia is a potentially dangerous complication, causing mortality in one of our patients. Therefore, when extracting this significant fact from our database in June 2010, we decided to commence primary closure of both mesenteric defects in all our patients.

Our team is currently performing 1,700 LRGB per year (Aleris Hospital Oslo and Aleris Obesitas Skåne), thus secondary closure of the defects in $11.1 \%$ of patients will demand a great deal of resources. By using the EndoHernia device, we did not have to change the placement of our trocar ports, and our operation time was only slightly increased (2-3 min).

Our study on primary closure is not without limitations. Mean time from primary operation without mesenteric closure to reoperation for internal hernia was 13 months. The herniafree survival diagram indicates that the incidence of clinically significant herniations continues from about 3 months post-op through at least the first 3 years. Follow-up time for the primary closure group is limited. In our view, it is very important at this stage to present our early observations and experience with the methodology as it is already gaining wide popularity. We will certainly report the results when follow-up duration has become appropriate for analysis.

However, the primary closures with our method have hitherto only resulted in four reoperations due to kinking of the enteroanastomosis and no mesenteric haematomas.

\section{Conclusion}

Our experience is in line with increasing evidence from several centres that the mesenteric defects should be closed primarily. The technique we describe is also very simple and safe. We cannot, however, at this moment, present long-term results on the efficacy of this closure method, but the results so far are promising.
Fig. 5 Jejunal mesenteric defect (left) and Petersen's defect (right) 11 months after closure with MS EndoHernia stapling device
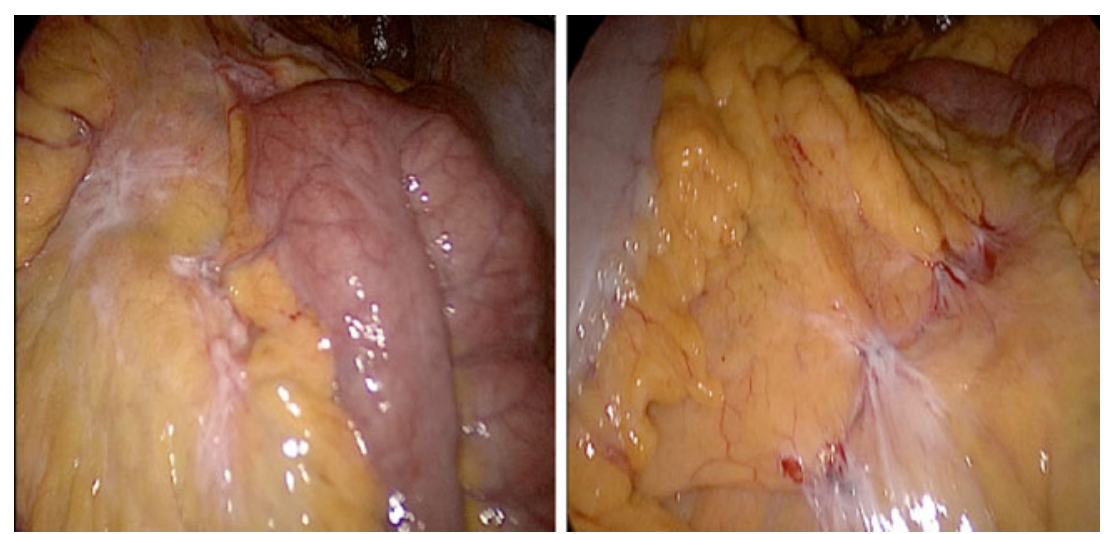
Acknowledgements We wish to acknowledge the nursing staff for entering data on basic patient demographics.

The present study was financed in part by the Crafoord Foundation.

Open Access This article is distributed under the terms of the Creative Commons Attribution Noncommercial License which permits any noncommercial use, distribution, and reproduction in any medium, provided the original author(s) and source are credited.

\section{References}

1. Leifsson BG, Gislason HG. Laparoscopic Roux-en-Y gastric bypass with 2-metre long biliopancreatic limb for morbid obesity: technique and experience with the first 150 patients. Obes Surg 2005; 15: 35-42.

2. Higa KD, Ho T, Boone KB. Internal hernias after laparoscopic Roux-en-Y gastric bypass: incidence, treatment and prevention. Obes Surg 2003; 13: 350-354.

3. Iannelli A, Buratti MS, Novellas S, Dahman M, Amor IB, Sejor E, et al. Internal hernia as a complication of laparoscopic Roux-en-Y gastric bypass. Obes Surg 2007; 17: 1283-1286.

4. Steele KE, Prokopowicz GP, Magnuson T, Lidor A, Schweitzer M. Laparoscopic antecolic Roux-en-Y gastric bypass with closure of internal defects leads to fewer internal hernias than the retrocolic approach. Surg Endosc 2008; 22: 2056-2061.

5. Champion JK, Williams M. Small bowel obstruction and internal hernias after laparoscopic Roux-en-Y gastric bypass. Obes Surg 2003; 13: 596-600.

6. Cho M, Pinto D, Carrodeguas L, Lascano C, Soto F, Whipple O, et al. Frequency and management of internal hernias after laparoscopic antecolic antegastric Roux-en-Y gastric bypass without division of the small bowel mesentery or closure of mesenteric defects: review of 1400 consecutive cases. Surg Obes Relat Dis 2006; 2: 87-91.

7. Quebbemann BB, Dallal RM. The orientation of the antecolic Roux limb markedly affects the incidence of internal hernias after laparoscopic gastric bypass. Obes Surg 2005; 15: 766770 .

8. Comeau E, Gagner M, Inabnet WB, Herron DM, Quinn TM, Pomp A. Symptomatic internal hernias after laparoscopic bariatric surgery. Surg Endosc 2005; 19: 34-39.

9. Gumbs AA, Duffy AJ, Chandwani R, Bell RL. Jejunojejunal anastomotic obstruction following laparoscopic Roux-en-Y gastric bypass due to non-absorbable suture: a report of seven cases. Obes Surg 2006; 16: 12-15.

10. Carmody B, DeMaria EJ, Jamal M, Johnson J, Carbonell A, Kellum J, et al. Internal hernia after laparoscopic Roux-en-Y gastric bypass. Surg Obes Relat Dis 2005; 1: 543-548. 